\title{
Primary Pulmonary Epithelial- Myoepithelial Tumour - Report of an Aggressive Clinical Case
}

\section{Authors \\ Dr Geethi M H', Dr Sajeed Abdul Rahuman², Dr Anila.K.R ${ }^{3}$}

${ }^{1}$ Senior Resident, Dept of Radiation Oncology, Regional Cancer Centre, Trivandrum 695011, Kerala, India

${ }^{2}$ Associate Professor, Dept of Radiation oncology, Regional Cancer Centre, Trivandrum, Kerala, India

${ }^{3}$ Assistant Professor, Dept of Pathology, Regional Cancer Centre, Trivandrum 695011, Kerala, India Corresponding Author

\section{Dr Sajeed Abdul Rahuman}

Associate Professor, Department of Radiation oncology, Regional Cancer Centre, Trivandrum 695011, India Email - sajerose@yahoo.com Phone: 919447041690

\begin{abstract}
Primary epithelial-myoepithelial tumor is usually a salivary gland neoplasm and extremely rare in the respiratory tract. These tumors are thought to arise from tracheo-bronchial submucosal glands. They usually present as early lesions due to the endobronchial location and have a benign course after surgical resection. In literature about twenty five cases have been reported so far and almost all of them had small tumors with a mean size of $2.5 \mathrm{~cm}$ with good prognosis after radical resection. We report a case with large unresectable primary pulmonary epithelial-myoepithelial tumour. The patient was a fifty eight year old gentleman who presented with a three week history of cough, chest pain, and breathlessness. CT scan of thorax showed a large heterogeneous mass lesion occupying upper right hemithorax infiltrating the great vessels. Trucut biopsy showed a neoplasm composed of cells arranged in diffuse sheets and glandular pattern with interspersed hyalinised material. Immunohistochemistry showed epithelial cells strongly positive for cytokeratin and myoepithelial cells positive for $p 63$. There was myoepithelial cell predominance and MIB 1 index was 7/10 HPF. Our case had an aggressive clinical course with poor response to chemotherapy and expired after nine months of diagnosis due to progressive disease. None of the reported cases had an extensive tumour with aggressive clinical behaviour as in our case.

Key words: Primary epithelial-myoepithelial tumor, aggressive
\end{abstract}

\section{INTRODUCTION}

An epithelial - myoepithelial tumor is an extremely rare pulmonary neoplasm. Usually these tumors occur in the salivary glands and represents approximately $1 \%$ of the primary tumors arising from them ${ }^{(1)}$. Tracheobronchial submucous glands are considered equivalent to the minor salivary glands and salivary gland like 
tumors can arise from these glands very rarely. Of these, epithelial - myoepithelial tumors are extremely rare and usually have a benign course after surgical resection. Usually the clinical symptoms start early as most are endobronchial with only a single reported parenchymal lesion to date ${ }^{(2)}$. Here, we report a case of a large unresectable pulmonary epithelial - myoepithelial tumor occupying upper right hemithorax with broad base towards mediastinum, encasing and compressing superior vena cava and infiltrating the great vessels with a short duration of symptom onset.

\section{CASE REPORT}

58 year old male with no significant medical or surgical history presented with cough, chest pain, breathlessness and hoarseness of voice of three weeks duration. He had a history of smoking one cigarette pack per day for past twenty years stopped one year back. Clinical evaluation revealed decreased breath sounds on right side. His chest X-ray showed homogenous opacity in the right hemithorax and USG chest showed lobulated mass lesion in right upper lobe with gross pleural effusion with total collapse of the underlying lung. His chest CT scan showed large heterogeneous mass lesion of size $16 \times 13 \times 11 \mathrm{~cm}$ in the right upper hemi thorax with broad base towards the mediastinum and anterior pleura. The interface of the tumor with the lung was well defined. The lesion was encasing and compressing the superior venacava with tumor thrombus extending to SVC and right atrium. The lesion completely encased and compressing right principle bronchus and upper lobar bronchus. The lesion was infiltrating the mediastinum and abutting the ascending aorta, arch of aorta, pulmonary trunk, right pulmonary artery and right superior pulmonary vein. The lesion was abutting the pleura for more than $5 \mathrm{~cm}$ with gross pleural effusion. No evidence of chest wall infiltration, bony erosion or lymphadenopathy noted (fig.1a and $1 b)$. His other routine investigations were all within normal range. A trucut biopsy was taken from the lung lesion which showed a neoplasm composed of cells arranged in diffuse sheets and focal glandular pattern with interspersed hyalinised material (fig.2a). Epithelial cells showed strong positivity for cytokeratin (fig.2b). Myoepithelial cells showed positivity for p63 (fig.2c). There was myoepithelial cell predominance and MIB-1 index was 7/10 HPF (IHCx400) (fig.2d).

A cardiology opinion was sought in view of tumor thrombus extending to right atrium. 2D echo showed good LV function, tumor infiltrating to atrial inflow causing compression. He was started on oral anticoagulation. Since the tumor was inoperable, he was started on palliative chemotherapy with Paclitaxel and Carboplatin. After four cycles, patient's clinical condition deteriorated and follow up CT taken 5 months after first CT (fig.1c and 1d) showed increase in size of the tumor $(18.5 \times 15.7 \times 16.8 \mathrm{~cm})$. The chemotherapy was changed to Vinorelbine on which he showed a mild symptomatic improvement. The disease progressed and the patient expired after 9 months of diagnosis. 


\section{JMSCR VoI||3||Issue||10||Page 7842-7846||October}

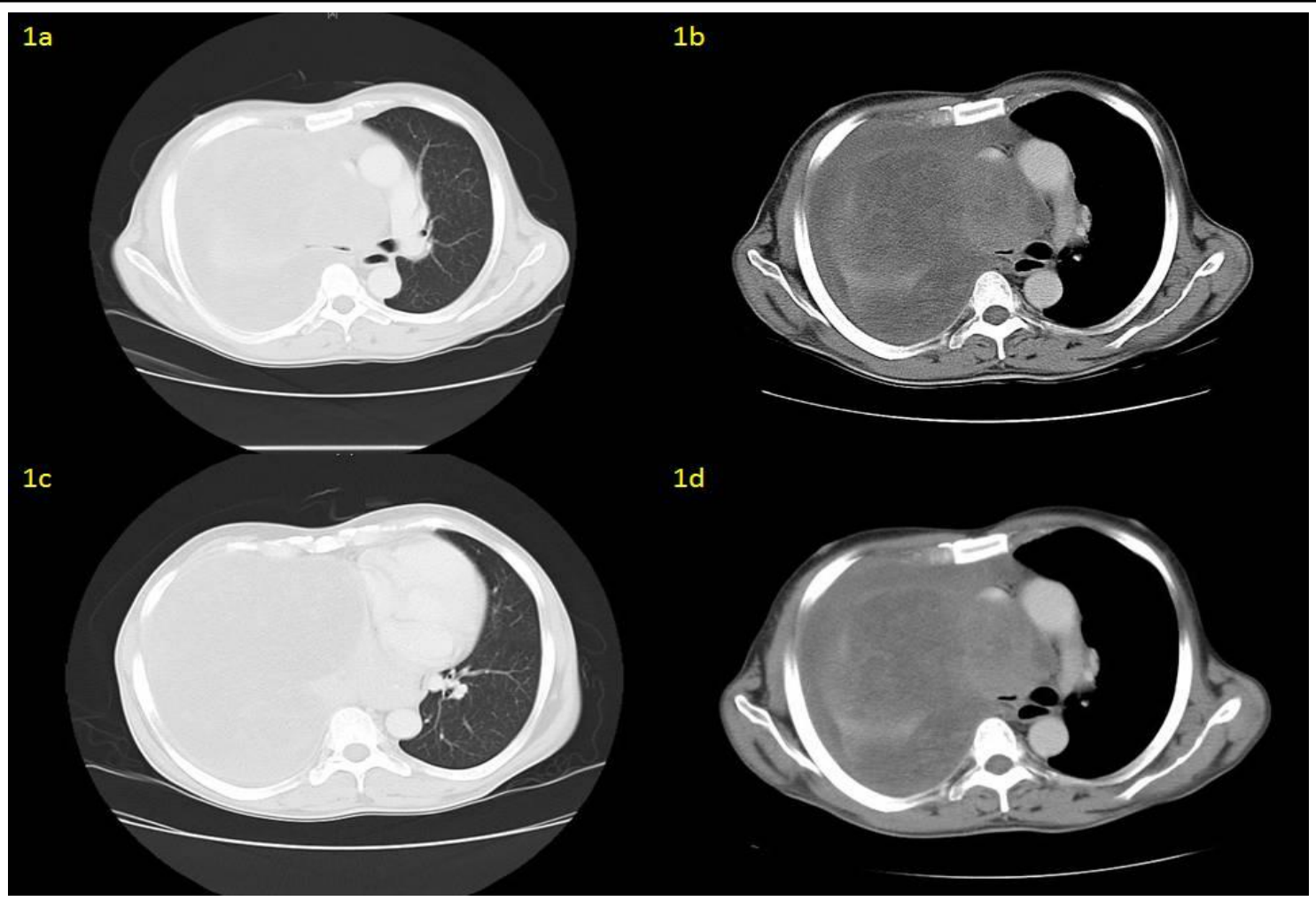

Fig. 1a and 1b: CT Thorax at initial presentation

Fig. 1c and 1d: CT Thorax after first line chemotherapy with four cycles of Paclitaxel and Carboplatin at 5 months.

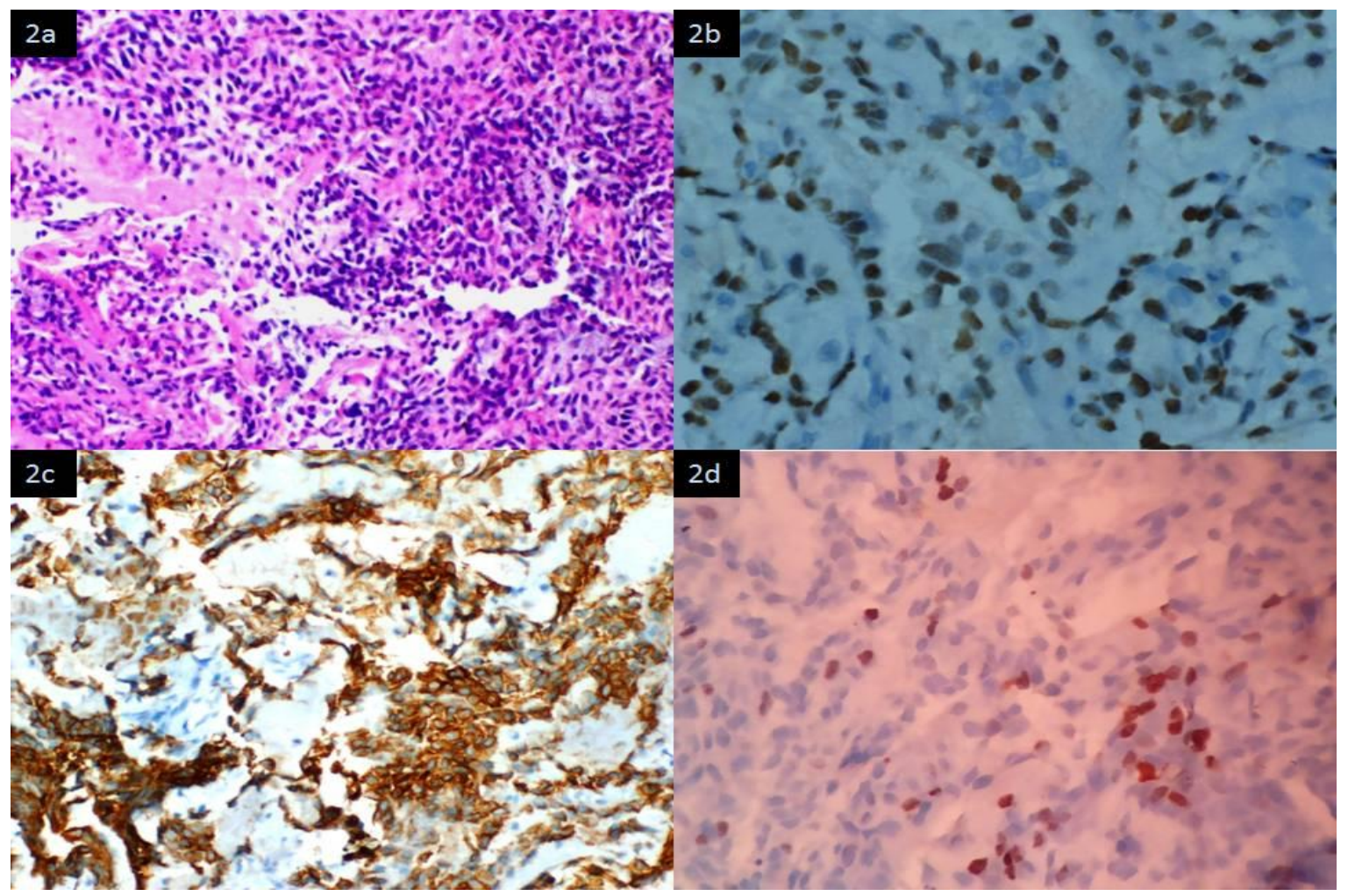

Fig. 2a: cells arranged in diffuse pattern and focal glandular pattern with interspersed hyalinised material (H\& E x 100).

Fig. 2b: Myoepithelial cells showing positivity for $\mathrm{p} 63$ (IHC x 400).

Fig. 2c: Epithelial cells showing strong positivity for cytokeratin (IHC x 200).

Fig. 2d: MIB-1 index 7/10 HPF (IHC x 400). 


\section{DISCUSSION}

Primary epithelial-myoepithelial tumors were first described in the salivary glands in $1972{ }^{(3)}$ with a lower incidence in the sweat glands and are extremely rare in the lungs. These rare tumors are thought to arise from the submucosal bronchial glands of the lower respiratory tract ${ }^{(4)}$, considered as a form of minor salivary glands. They develop mostly in the proximal bronchial tree, usually as polypoid endobronchial lesions. The WHO defines this entity as "a malignant tumor composed of variable proportions of two cell types, which typically form duct like structures. The biphasic morphology is represented by an inner layer of duct lining, epithelial type cells and an outer layer of clear myoepithelial cells" (1). Pulmonary epithelial myoepithelial tumors are usually of small size when detected, accounting for the early symptomatology due to their endobronchial location. Farzana Arif et al (5) reviewed the literature from reports of 25 cases and have shown that this tumor usually occurs in middle aged patients with a slight female predilection (F: M-1.5:1), most cases presented as polypoid endobronchial lesions. The tumor size of the 23 available cases reviewed ranged from 0.8 $\mathrm{cm}$ to $5 \mathrm{~cm}$, with a median value of $2.5 \mathrm{~cm}$. In our case the patient had a short history of three weeks respiratory symptoms at presentation and the tumor was $16 \mathrm{~cm}$ which was much larger than any of the reported cases. Usually these tumors will be well delineated masses on gross examination though not well encapsulated ${ }^{(2)}$. But in our case the patient presented in an advanced stage with an unresectable tumor invading the mediastinal structures. Tumors described as predominantly myoepitheliomatous are aggressive and have a high mitotic rate. Presence of tumor necrosis and nuclear pleomorphism are typically adverse prognostic factors ${ }^{(6,7)}$. In our case there was myoepithelial predominance and the MIB-1 index was 7/10 HPF (IHCx400). These tumors are usually low grade tumors responding well to surgical excision ${ }^{(8,9)}$. Almost all reported cases had resectable lesions and had event free survival after surgery. In our patient, the tumor was unresectable and response to chemotherapy as well as survival was also poor. In the report of five cases by Nguyen et al ${ }^{(10)}$, one case showed extensive lymphovascular perineural invasion and peribronchial lymphnode metastases. None of the previous case reports showed an aggressive presentation as in our case.

\section{CONCLUSION}

Epithelial myoepithelial tumors are rare type of pulmonary tumors which pathologically shows biphasic morphology. Our case presented with an inoperable advanced lesion, had an aggressive clinical course and a poor outcome when most of the reported cases showed well delineated endobronchial lesions with good response to surgical resection.

\section{Acknowledgements: Nil}

\section{REFERENCES}

1. Fonseca and J. Soares. "Epithelial myoepithelial carcinoma,"in World Health Organization Classification of Tumors, Pathology and Genetics of Head and Neck Tumors. L. Barnes, J. W. Eveson, P. Reichart, and D. Sidransky, Eds. pp. 225226, IARC Press, Lyon, France, 2005

2. G. Munoz, F. Felipo, I. Marquina, and C. Del Agua. Epithelial-myoepithelial tumour of the lung: a case report referring to its molecular histogenesis. Diagnostic Pathology, vol. 6, article 71, 2011.

3. Donath K, Seifert G, Schmitz R. Diagnosis and ultrastructure of the tubular carcinoma of salivary gland ducts: epithelialmyoepithelial carcinoma of the intercalated ducts [in German]. Virchows Arch A Pathol Pathol Anat. 1972; 356:1631.

4. Moran CA. Primary salivary gland-type tumors of the lung. Semin Diagn Pathol. 1995.12:106-22. 
5. Farzana Arif, Susan Wu, Shahriyour Andaz, and Stewart Fox. "Primary Epithelial Myoepithelial Carcinoma of Lung, Reporting of a Rare Entity, Its Molecular Histogenesis and Review of the Literature," Case Reports in Pathology. vol. 2012, Article ID 319434, 6 pages, 2012. doi:10.1155/2012/319434.

6. L. G. Fulford, Y. Kamata, K. Okudera et al. "Epithelial myoepithelial carcinomas of the bronchus," The American Journal of Surgical Pathology. vol. 25, no. 12, pp. 1508-1514,2001.

7. Ru Kun, Srivastava A, Tischler, AS. Bronchial epithelial myoepithelial carcinoma. Arch Pathol Lab Med. 2004.128:92-4.

8. Wilson RW, Moran CA. Epithelialmyoepithelial carcinoma of the lung: immunohistochemical and ultrastructural observations and review of the literature. Hum Pathol. 1997;28:631-5.

9. Doganay L., Bilgi S., Ozdil A., Yoruk Y., Altaner S., Kutlu K. Epithelialmyoepithelial carcinoma of the lung: a case report and review of literature. Arch Pathol Lab Med. 2003;127(4):177-180.

10. C. V. Nguyen, S. Suster, and C. A. Moran, "Pulmonary epithelial-myoepithelial carcinoma: a clinicopathologic and immunohistochemical study of 5 cases," Human Pathology, vol. 40, no. 3, pp. 366373, 2009. 